Rev. Adm. Saúde - Vol. 18, № 72, jul. - set. 2018

http://dx.doi.org/10.23973/ras.72.126

ARTIGO ORIGINAL

\title{
Estudo de custo-benefício na utilização de campos / kits cirúrgicos têxteis $\mathrm{x}$ campos / kits descartáveis estéreis
}

Cost-benefit study on the use of textile surgical fields / kits $x$ sterile disposable fields / kits

\section{Lucas de Oliveira Freitas Moraes', Fátima Guimarães Fraga², Paulo Ernesto Malard Assad ${ }^{3}$, Sônia Maria Nunes Viana ${ }^{4}$, Keli Bahia Felicíssimo Zocratto $^{5}$}

1. Graduando em gestão de serviços de saúde da Universidade Federal de Minas Gerais (UFMG), Belo Horizonte MG

2. Pedagoga, especialista em gestão de qualidade hospitalar. Chefe da Unidade de Governança e Higienização do Hospital das Clínicas da UFMG, Belo Horizonte, MG

3. Administrador, especialista em gestão da qualidade hospitalar. Administrador do Hospital das Clínicas da UFMG, Belo Horizonte MG

4. Enfermeira, mestre em enfermagem. Professora da Escola de Enfermagem da UFMG, Belo Horizonte MG

5. Cirurgiã-dentista, doutora em saúde pública. Professora adjunta da Escola de Enfermagem da UFMG, Belo Horizonte MG

\section{RESUMO}

A gestão de custos nas organizações de saúde principalmente no Sistema Único de Saúde (SUS) passou a ser um exercício fundamental com a necessidade de assegurar melhor eficiência na aplicação dos recursos e sustentabilidade do sistema. Este estudo foi realizado na Unidade de Governança e Higienização em um hospital universitário em Minas Gerais. Teve como objetivos calcular o custo geral para o reprocessamento dos campos cirúrgicos têxteis e comparar com os valores entre campos cirúrgicos descartáveis estéreis disponíveis no mercado. Para os cálculos, foram 
coletadas informações relativas às despesas número de pessoal necessário para o recebimento e o acondicionamento dos pacotes, esterilização, estocagem, distribuição dos pacotes de campos cirúrgicos, salários, encargos sociais, benefícios dos profissionais envolvidos no reprocessamento dos campos, quantidade e custos dos insumos utilizados no preparo dos campos, dos materiais consumidos no processo de esterilização (água, vapor e energia elétrica) e serviços terceirizados que participam do custo final do reprocessamento. Os campos descartáveis obtiveram uma redução do custo quando comparados aos têxteis. Ao analisar a produção diária do CME (Centro de Materiais e Esterilização) de acordo com a demanda do hospital em estudo, a redução dos custos se torna ainda maior.

Palavras-chave: Análise de custos; campos cirúrgicos; processamento; esterilização

\begin{abstract}
The management of health in health organizations, especially in the Unified Health System (SUS), has become fundamentally fundamental for conducting research on the resources and sustainability of the system. This study was carried out at the Governance and Hygiene Unit at a university hospital in Minas Gerais. It aimed to calculate the overall cost for the reprocessing of the textile surgical fields and to compare it with the values between sterile disposable surgical fields available in the market. For the calculations, information was collected regarding the number of personnel required to receive and package packages, sterilization, storage, distribution of surgical field packages, salaries, social charges, benefits of professionals involved in reprocessing fields, quantity and costs of inputs used in field preparation, materials consumed in the sterilization process (water, steam and electricity) and outsourced services that participate in the final cost of reprocessing. The disposable fields obtained a reduction of the cost for the hospital when compared to the textiles. When analyzing the daily production of the CME (Material Center and Sterilization) according to the demand of the hospital under study, the reduction of costs becomes even greater.
\end{abstract}

Keywords: Cost analysis; surgical fields; processing; sterilization

\title{
INTRODUÇÃO
}

As práticas relacionadas aos cuidados à saúde exigem o uso de aventais e campos cirúrgicos para evitar a contaminação por agentes infecciosos. A paramentação cirúrgica é composta por campos cirúrgicos estéreis com propósito de impedir a passagem de microorganismos da equipe cirúrgica e do 
paciente para o campo operatório, diminuindo os riscos relacionados à contaminação e infecção. Existem duas variedades de aventais e campos cirúrgicos: os de tecido reutilizáveis e os descartáveis. Os primeiros percorrem o processo de lavagem, secagem, empacotamento e esterilização após seu uso, devendo ser monitorados em relação ao número de lavagens a que são submetidos. Os outros, considerados descartáveis, são confeccionados com material SMS ou TNT e encontram-se disponíveis no mercado já esterilizado e para pronto uso, sendo utilizados apenas uma vez ${ }^{1}$.

No mercado também existem kits específicos para cada clínica cirúrgica e kits cirúrgicos universais descartáveis estéreis, que compõe em média 6 campos cirúrgicos. Conforme foi informado pelas equipes cirúrgicas e enfermeiros do centro de material e esterilização do hospital em estudo, na prática clínica o pacote de um kit cirúrgico universal e kits específicos para cada clínica pode não atender completamente à especificidade de cada cirurgia realizada, sendo sua utilização variável de acordo com o porte e a demanda dos procedimentos realizados. Portanto não é incomum, de acordo com a especialidade cirúrgica, o uso de mais kits universais e específicos apenas para o uso de um campo, ocorrendo o desperdício e, consequentemente, o aumento do custo do processo. Com essa preocupação de desperdício, o hospital adaptou seus kits cirúrgicos têxteis de acordo com as suas necessidades cirúrgicas para cada clínica.

A gestão de custos no Sistema Único de Saúde (SUS) tornou-se exercício fundamental com a necessidade de melhorar a eficiência na aplicação dos recursos. É importante destacar que os custos são geridos objetivando ao corte ou à redução de forma precisa. O Ministério da Saúde, o Conselho Nacional de Secretários de Saúde (Conass) e o Conselho Nacional de Secretarias Municipais de Saúde (Conasems) estabeleceram métodos e ferramentas que possibilitem a otimização de custos e que possam calcular os recursos financeiros para o custeio global do SUS. Este reconhecimento demonstra a importância da gestão de custos para as estratégias dos gestores dos serviços de saúde ${ }^{2}$.

A instituição em estudo trata-se de um hospital universitário, público e geral que realiza atividades de ensino, pesquisa e assistência, sendo referência no sistema municipal e estadual de saúde no atendimento aos pacientes portadores de patologias de média e alta complexidade. É campo de ensino para os cursos de medicina, enfermagem, farmácia, fisioterapia, odontologia, terapia ocupacional, psicologia, nutrição, fonoaudiologia, gestão de serviços de saúde e tecnologia em radiologia. A instituição sempre realizou a aquisição, confecção, processamento, triagem, empacotamento e esterilização dos campos têxteis. A chefia responsável pelo setor está nesta função há mais de dez anos e apresenta domínio dos processos como um todo, das suas necessidades de adequação para aperfeiçoa-los e atualmente trabalha com uma equipe bem estruturada. Exercita bem a articulação na rede de contratualidade entre as unidades de serviços da instituição.

Neste sentido, o presente estudo teve como objetivo calcular os custos para o reprocessamento dos campos cirúrgicos têxteis e comparar os valores entre 0 
custo geral do processamento dos campos têxteis estéreis com campos cirúrgicos estéreis descartáveis, disponíveis no mercado.

\section{MATERIAL E MÉTODOS}

O presente estudo, de cunho descritivo e desenho transversal, foi realizado durante o período de maio a julho de 2018, na Unidade de Governança e Higienização de um hospital público universitário no Estado de Minas Gerais. A instituição governamental, também voltada ao ensino, possui aproximadamente 500 leitos e presta assistência aos pacientes do Sistema Único de Saúde (SUS). A Unidade de Governança e Higienização do referido hospital é composta pela lavanderia, sala de costura e empresa de higienização hospitalar.

A amostra do estudo foi constituída por um grupo referência composto por kits de campos cirúrgicos de material têxtil (100\% algodão) com dimensões e pesos específicos e um grupo de comparação composto por campos cirúrgicos de material descartável seguindo as mesmas dimensões estabelecidas pelos kits de referência. Para a composição do grupo de referência utilizaram-se inicialmente, quatro campos cirúrgicos de material têxtil com dimensões e pesos específicos que, posteriormente, foram combinados entre si ou individualmente para a composição de oito kits cirúrgicos (grupo de referência). A escolha dos itens que compõem cada kit considerou as necessidades cirúrgicas específicas de cada clínica do hospital e, para a composição dos oito kits foram utilizados, ao total, 39 campos cirúrgicos. Os kits descartáveis foram criados seguindo a mesma distribuição de campos cirúrgicos existentes no respectivo kit de comparação (grupo de referência). Para definir o preço final de cada kit descartável, realizou-se a soma de preços individuais de cada campo cirúrgico descartável utilizado. Kits universais e cirúrgicos de especialidades encontrados no mercado não foram considerados devido a seus valores elevados e pela particularidade das composições do kit cirúrgico que o hospital em análise utiliza. A distribuição dos kits cirúrgicos segundo quantidade e dimensão de campos cirúrgicos utilizados encontra-se no Quadro 1.

Quadro 1. Distribuição dos kits cirúrgicos segundo quantidade e dimensão dos campos cirúrgicos utilizados.

\begin{tabular}{|l|r|r|r|r|r|r|r|r|}
\hline Campos cirúrgicos & \multicolumn{7}{|l|}{ Tipos de kits/quantitativos de campos } \\
\hline $\begin{array}{l}\text { Medida (m) e peso } \\
\text { (kg) }\end{array}$ & Kit A & \multicolumn{1}{|l|}{ Kit B } & \multicolumn{1}{|l|}{ Kit C } & \multicolumn{1}{l|}{ Kit D } & Kit E & Kit F & Kit G & Kit H \\
\hline $0,80 \times 0,80(0,160)$ & 0 & 1 & 0 & 0 & 1 & 0 & 1 & 0 \\
\hline $1,00 \times 1,00(0,765)$ & 0 & 1 & 2 & 0 & 0 & 4 & 1 & 0 \\
\hline $1,00 \times 1,00(0,291)$ & 0 & 3 & 2 & 0 & 2 & 0 & 3 & 3 \\
\hline
\end{tabular}




\begin{tabular}{|l|r|r|r|r|r|r|r|r|}
\hline $1,40 \times 1,60(0,506)$ & 6 & 0 & 2 & 7 & 5 & 0 & 0 & 1 \\
\hline
\end{tabular}

Legenda: Kit $\mathrm{A}$ (kit cirúrgico). Kit $\mathrm{B}$ (kit pi). Kit $\mathrm{C}$ (kit parto). Kit $\mathrm{D}$ (kit bo). Kit $\mathrm{E}$ (kit hemodinâmica). Kit $F$ (kit farmacotécnico). Kit G (kit experimental). Kit $\mathrm{H}$ (kit odonto).

Para o cálculo dos custos envolvendo cada pacote de campo cirúrgico têxtil (100\% algodão) foi necessária a busca de dados em diversos setores envolvidos com o processo em análise: preço de aquisição dos campos e insumos (fita-crepe, fita adesiva, indicador químico e biológico, etiqueta de identificação, gorro e máscara) utilizados no processamento dos campos têxteis $100 \%$ algodão (dados obtidos no almoxarifado - Sistema Net Term); preço do quilograma de vapor produzido e da energia elétrica para a produção do vapor e para o funcionamento da autoclave (dado obtido na unidade de engenharia hospitalar - UEH); quantidade diária de campos cirúrgicos têxteis esterilizados (dados obtidos no Centro de Material e Esterilização); preço da água e do esgoto (dado obtido no serviço de infraestrutura física - SIF); salários, benefícios e encargos sociais dos profissionais envolvidos nos processos em análise (setor de custos - SC); preço do quilograma de roupa lavada em lavanderia terceirizada.

Para o cálculo dos custos relacionados ao processamento dos campos e kits têxteis (100\% algodão) foi utilizado: o custo de empacotamento considerando a quantidade do campo cirúrgico específico que caberia em uma rack (peso do campo + tamanho do SMS utilizado + fita branca + fita para autoclave); o custo da esterilização (teste Bowe Dick + teste desafio + integrador químico); a mão de obra utilizada na esterilização (tempo total de ciclo x custo por minuto da mão de obra); a mão de obra do preparo (custo do tempo de triagem de um hamper + tempo de empacotamento de um hamper / quantidade de campos no hamper); o custo indireto por pacote (custo da limpeza + telefonia + central de equipamentos-horas/utilizadas + almoxarifado-valor fornecido / produção); os custo diretos (custo de serviços externos + manutenção + energia / produção); custo da caldeira (custo por ciclo do vapor saturado / pacotes de campos por ciclo); custo do descarte (peso em gramas da folha utilizada $x$ custo do descarte / 1000). O custo total por item (custo da esterilização + mão de obra da esterilização / quantidade de campos + custo do empacotamento + mão de obra de preparo) e o preço final (total por item + custo direto + custo indireto + custo da caldeira + custo do descarte) foram calculados considerando as variáveis acima.

Para o cálculo do valor de compra dos campos descartáveis estéreis foi realizada uma consulta no mercado com seis empresas que atendem as exigências e especificidades estabelecidas pelo hospital em referência. Após o orçamento dos campos, os preços estabelecidos basearam-se na média dos valores encontrados no mercado. Os valores são apresentados em moeda nacional (R\$) e respectiva mensuração em dólar americano (US\$). Ressalta-se que os kits descartáveis foram construídos seguindo a mesma distribuição de produtos do kit têxtil. 


\section{RESULTADOS}

A comparação dos gastos do reprocessamento dos campos cirúrgicos têxteis com os descartáveis mostrou um aumento proporcional dos gastos considerando o aumento da dimensão do campo cirúrgico, independente se têxtil ou descartável. Observou-se também que, independentemente do tamanho do campo, invariavelmente, o custo do material têxtil foi maior que o descartável (Tabela 1). Ressalta-se que os custos do campo têxtil ( $100 \%$ algodão) foi calculado considerando toda trajetória do processamento e cálculos necessários para custeá-los após lavagem e esterilização.

Tabela 1. Gastos com reprocessamento dos campos cirúrgicos têxteis $x$ campos descartáveis estéreis (US\$1,00 $=R \$ 3,94)$.

\begin{tabular}{lrrr}
\hline Campos cirúrgicos & $\begin{array}{l}\text { Preço têxtil R\$ } \\
\text { (US\$) }\end{array}$ & $\begin{array}{l}\text { Preço de mercado } \\
\text { descartável } \\
\text { estéril R\$ (US\$) }\end{array}$ & $\begin{array}{l}\text { Diferença de } \\
\text { preço R\$ (US\$) }\end{array}$ \\
\hline $0,80 \times 0,80$ & $7,14(1,85)$ & $5,65(1,46)$ & $1,49(0,39)$ \\
$1,00 \times 1,00$ (duplo) & $11,19(2,90)$ & $9,20(2,38)$ & $1,99(0,51)$ \\
$1,00 \times 1,00$ & $7,78(2,01)$ & $4,80(1,24)$ & $2,98(0,77)$ \\
$1,40 \times 1,60$ & $9,35(2,42)$ & $9,55(2,47)$ & $0,20(0,05)$ \\
\hline Total & $\mathbf{3 5 , 4 6 ( 9 , 1 7 )}$ & $\mathbf{2 9 , 2 0 ( 7 , 5 5 )}$ & $\mathbf{6 , 6 6 ( 1 , 7 2 )}$ \\
\hline
\end{tabular}

Ao se comparar o custo para o reprocessamento dos kits cirúrgicos têxteis $100 \%$ algodão com o custo dos kits cirúrgicos descartáveis estéreis observouse que, na maioria dos kits analisados, os custos envolvidos com os descartáveis foram menores. Apenas o campo cirúrgico 1,40 x 1,60 apresentou um preço maior para o descartável. A diferença de custos entre os kits têxteis e descartáveis variou entre $R \$ 0,20$ a $R \$ 2,98$ sendo que as maiores diferenças foram observadas quando o descartável tinha preço menor ao têxtil (Tabela 2).

Tabela 2. Gastos com kits específicos de cada clínica do hospital em análise e o custo dos kits descartáveis (US\$1,00 $=\mathrm{R} \$ 3,94$ ).

\begin{tabular}{lrrr}
\hline Kits cirúrgicos & $\begin{array}{l}\text { Preço têxtil R\$ } \\
\text { (US\$) }\end{array}$ & $\begin{array}{l}\text { Preço de mercado } \\
\text { descartável R\$ } \\
\text { (US\$) }\end{array}$ & $\begin{array}{l}\text { Diferença de } \\
\text { preço R\$ (US\$) }\end{array}$ \\
\hline Cirúrgico & $60,59(15,68)$ & $57,30(14,83)$ & $3,29(0,85)$ \\
$\mathrm{Pi}$ & $46,09(11,93)$ & $20,05(5,19)$ & $26,04(6,73)$ \\
Parto & $61,15(15,82)$ & $47,10(12,18)$ & $14,05(3,63)$ \\
Bo & $69,93(18,10)$ & $66,85(17,29)$ & $3,08(0,80)$ \\
Hemodinâmica & $73,94(19,13)$ & $63,00(16,29)$ & $10,94(2,83)$ \\
Farmacotécnico & $49,28(12,75)$ & $36,80(9,52)$ & $12,48(3,23)$ \\
\hline
\end{tabular}




\begin{tabular}{|c|c|c|c|}
\hline Experimental & $46,09(11,93)$ & $29,25(7,56)$ & $16,84(4,35)$ \\
\hline Odonto & $37,10(9,60)$ & $23,95(6,19)$ & $13,15(3,40)$ \\
\hline Total & $444,17(115,00)$ & $344,30(89,04)$ & $99,87(25,83)$ \\
\hline
\end{tabular}

Considerando a produção diária do CME do Hospital em referência em relação aos Kits cirúrgicos específicos de cada clínica, observou-se que os kits cirúrgico e Pi foram os que demandam maior produção, seguidos pelos kits da hemodinâmica e do parto. Os de menor produção diária foram o experimental, farmacotécnico, Bo e odontologia (Tabela 3). Ao comparar os custos dos kits têxteis e descartáveis segundo a produção diária, observou-se que, em sua maioria, os kits descartáveis apresentaram menores custos. Observou-se uma redução dos custos de $R \$ 2.249,95$ ao se comparar o uso dos kits descartáveis com os têxteis (Tabela 3).

Tabela 3. Gastos com produção diária do CME com kits cirúrgicos têxteis $x$ descartáveis estéreis (US\$1,00 = R \$3,94).

\begin{tabular}{|c|c|c|c|c|}
\hline Kits cirúrgicos & $\begin{array}{l}\text { Produção } \\
\text { diária }\end{array}$ & $\begin{array}{l}\text { Kits têxteis } \\
\text { R\$ (US\$) }\end{array}$ & $\begin{array}{l}\text { Kits descartáveis } \\
\text { R\$ (US\$) }\end{array}$ & $\begin{array}{l}\text { Diferença de } \\
\text { preço R\$ (US\$) }\end{array}$ \\
\hline Cirúrgico & 60 & 3635,55 (94) & $3438,00(889)$ & $197,55(51,09)$ \\
\hline $\mathrm{Pi}$ & 60 & $2765,59(715)$ & $1203,00(311)$ & $1562,59(404)$ \\
\hline Parto & 12 & $733,82(190)$ & $565,20(146)$ & $168,62(43,45)$ \\
\hline Bo & 5 & $349,67(90,43)$ & $334,25(86,44)$ & $5,42(1,40)$ \\
\hline Hemodinâmica & 14 & $1035,23(268)$ & $882,00(228)$ & $153,23(39,63)$ \\
\hline Farmacotécnico & 4 & $197,12(50,98)$ & $147,20(38,07)$ & $49,92(12,91)$ \\
\hline Experimental & 2 & $92,19(23,84)$ & $58,50(15,13)$ & $33,69(8,71)$ \\
\hline Odonto & 6 & $222,63(57,57)$ & $143,70(37,10)$ & $78,93(20,41)$ \\
\hline Total & 163 & $9031,80(2336)$ & $6771,85(1751)$ & $2249,95(582)$ \\
\hline
\end{tabular}

Os campos individuais descartáveis obtiveram uma redução de $R \$ 6,26$ quando comparados aos têxteis. Os Kits descartáveis compostos pelo valor de cada campo descartável estéril individual obtiveram uma redução de $R \$ 99,87$. Ao analisar produção diária do CME de acordo com a demanda do hospital em estudo, a redução dos custos se torna ainda maior, enfatizando a vantagem financeira com o uso de campos descartáveis, reduzindo o custo de $\mathrm{R} \$$ $9.031,80$ para $\mathrm{R} \$ 6.771,85$.

Em relação aos resíduos produzidos, a instituição em estudo paga $R \$ 2,13$ o quilograma para o descarte de resíduos químicos/biológicos e $R \$ 0,01$ para resíduos comuns como as embalagens que envolvem os campos descartáveis. Os custos para o descarte tanto dos campos têxteis e campos descartáveis já estão inseridos no preço final de cada campo e kits cirúrgicos. O custo da esterilização e descarte de cada Kit se encontram no Quadro 2. 
Quadro 2. Custo da esterilização/descarte de cada kit cirúrgico, em real e dólar americano (US\$1,00 = R\$3,94).

\begin{tabular}{|l|r|r|r|r|r|r|r|r|}
\hline $\begin{array}{l}\text { Custo da } \\
\text { esterilização } \\
\text { ldescarte }\end{array}$ & Kit A & Kit B & Kit C & Kit D & Kit E & Kit F & Kit G & Kit H \\
\hline R\$ & 4,49 & 4,42 & 4,52 & 4,51 & 4,51 & 4,51 & 4,41 & 4,41 \\
\hline US\$ & 1,12 & 1,11 & 1,13 & 1,13 & 1,13 & 1,13 & 1,10 & 1,10 \\
\hline
\end{tabular}

\section{DISCUSSÃO}

Os custos relacionados ao uso de kits têxteis foram maiores, em sua maioria, quando comparados aos custos dos kits descartáveis. O reprocessamento de kits têxteis necessita da atuação de diversos setores no hospital envolvendo custos diretos e indiretos abrangendo gastos com o pessoal, infraestrutura, equipamentos e insumos, dentre outros. A perspectiva de redução dos gastos a partir da possibilidade de se reutilizar os kits têxteis por meio do reprocessamento parece não se sustentar em todas as situações, uma vez que, na maioria das condições analisadas, houve maior custo envolvendo esses kits.

Uma variável que diferencia significativamente os dois grupos analisados se refere à descartabilidade e reprocessamento dos materiais o que gera impactos distintos nos custos envolvendo o uso dos kits. No caso de kits têxteis, custos envolvendo transporte interno, processamento (lavagem, secagem, empacotamento e esterilização) e pessoal estão intimamente relacionadas ao aumento dos gastos. Os campos cirúrgicos têxteis têm um tempo de vida útil estipulado, uma vez que o número de reprocessamento do material deve ser controlado pelo setor responsável. Campos duplos podem ser reprocessados por até 65 vezes e os campos simples apenas 5 vezes $^{3}$. A legislação também ressalta que não é permitido uso de campos têxteis $100 \%$ algodão com remendos, perfurações, rasgos e desgaste que possam comprometer a barreira microbiana do campo em uso ${ }^{4}$. O campo têxtil $100 \%$ algodão foi o mais utilizado mundialmente nos hospitais até o surgimento dos campos descartáveis estéreis. Mesmo com o surgimento dos campos descartáveis o campo de tecido ainda é muito utilizado nas instituições hospitalares brasileiras 5 .

No caso dos kits descartáveis, uma vez adquiridos, os mesmos apresentam um fluxo interno unidirecional até o seu uso e posterior descarte. Locais que geram muitos resíduos como o bloco cirúrgico, por exemplo, que é composto pelo centro cirúrgico e pelo centro de material e esterilização, devem ser orientados em todas as etapas de gerenciamento de resíduos visando sua redução e 
consequentemente dos custos envolvidos. O uso de kits descartáveis gera aumento no custo de resíduos pois serão produzidos resíduos químico/biológico e resíduos comuns pelo descarte da embalagem. A geração de resíduos é inevitável e aumenta significativamente com o uso de descartáveis e, neste sentido, a implementação de um plano para separação e diminuição de resíduos sólidos de saúde nos diversos setores, que requer a participação de todas as equipes para o correto descarte, que deve ter seu destino adequado ${ }^{6}$.

Os campos descartáveis são de material SMS compostos por fibras contínuas e denso aglomerado de microfibras tortuosas que agem como barreira contra agentes contaminantes ou TNT, composto por fibras ou filamentos naturais, artificiais ou sintéticos ${ }^{7}$. Diferencia-se do tecido convencional unicamente pela forma que suas linhas são tecidas, fato este que definirá a gramatura do tecido ${ }^{8}$.

Essas características respaldam o uso do kit descartável, uma vez que resguardam os princípios básicos da segurança exigida na prática da assistência em saúde. A segurança aliada a um melhor custo-benefício evidencia ser razoável a escolha dos kits descartáveis uma vez que estes proporcionam uma redução dos custos vinculados à produção diária em aproximadamente $30 \%$.

O hospital em referência mantém um controle de cada campo cirúrgico têxtil em relação ao número de reprocesso. No entanto, mesmo a instituição obtendo controle deste número, tendo uma equipe qualificada e treinada para cada etapa dos campos de tecido, a gestão de custos do hospital deve considerar a eficiência dos processos principalmente por tratar-se de uma instituição pública, com recursos financeiros escassos. Porém, o estudo dos custos em instituições hospitalares é um trabalho bastante complexo, devido a diversidade de serviços prestados e que exige a delimitação dos centros de custos e um eficiente sistema de informações ${ }^{9}$.

\section{CONSIDERAÇÕES FINAIS}

Os campos cirúrgicos individuais descartáveis estéreis apresentaram o melhor custo-benefício em relação aos campos têxteis 100\% algodão sendo possível perceber que, do ponto de vista financeiro, os campos descartáveis estéreis são mais vantajosos. Apesar de gerarem maior quantidade de resíduos, os kits descartáveis são uma alternativa bastante viável a partir do momento em que existe a conscientização e envolvimento dos setores no gerenciamento de resíduos das diversas unidades do hospital. Outro fator que torna razoável a escolha dos kits descartáveis é a redução dos custos relacionados à produção diária dos kits gerando uma economia em torno de $30 \%$. No entanto, cabe ressaltar a importância de análises de custos voltadas para a realidade de cada instituição, principalmente pela sua demanda diária com os campos e Kits cirúrgicos fazendo com que a análise de custos se torne um processo dinâmico e atualizado regularmente. 


\section{REFERÊNCIAS}

1. Burgatti JC, Lacerda RA. Revisão Sistemática sobre aventais cirúrgicos no controle da contaminação/infecção do sítio cirúrgico. Rev Esc Enferm USP 2009; 43(1):237-44.

2. Ministério da Saúde. Organização Pan-Americana da Saúde. Introdução à Gestão de Custos em Saúde; 2013. Disponível em: < www.saude.gov.br/bvs >.

3. Agência Nacional de Vigilância Sanitária (Brasil). Resolução RDC. RDC no 15, de 15 de março de 2012.

4. Burgatti JC, Possari JF, Moderno AMB. Avaliação da barreira microbiana do campo cirúrgico simples do algodão. Rev SOBECC 2004; 9(1): 24-32.

5. Fernandes AT. Infecção hospitalar e suas interfaces na área da saúde. Vol. 1. São Paulo: Atheneu, 2000. p. 491, 493-494.

6. Agência Nacional de Vigilância Sanitária (Brasil). Resolução da Diretoria Colegiada RDC no 33, de 25 de fevereiro de 2003. Dispõe sobre o Regulamento Técnico para o gerenciamento de resíduos de serviços de saúde. Diário Oficial da República Federativa do Brasil, Brasília (DF). [Internet] [citado 2003 Dez 10]. Disponível em: <http://www.anvisa.gov.br>.

7. Gouveia VR. Embalagens para esterilização: tecidos e não tecidos. Rev. SOBECC 1997; 2(4):10-2.

8. Associação Brasileira de Normas Técnicas. ABNT NBR 14767: Artigos têxteis hospitalares - Compressa campo operatório - Requisitos e métodos de ensaio. Rio de Janeiro: ABNT; 2009.

9. Bonacim CAG, Araujo AMP. Gestão de custos aplicada a hospitais universitários públicos: a experiência do Hospital das Clínicas da Faculdade de Medicina de Ribeirão Preto da USP. Rev. Adm. Pública 2010; 44(4), 90331.

Recebido: 24 de agosto de 2018. Publicado: 20 de setembro de 2018 .

Correspondência: Keli Bahia Felicíssimo Zocratto. E-mail:

kelibahia@yahoo.com.br

Conflito de Interesses: os autores declararam não haver conflito de interesses. 
(C) This is an Open Access article distributed under the terms of the Creative Commons Attribution License, which permits unrestricted use, distribution, and reproduction in any medium, provided the original work is properly cited 